\title{
A mathematical model for chemo-immunotherapy of cancer considering macrophages polarization and cytokine dynamics
}

\author{
Negin Darandis ${ }^{1}$ and Mostafa Nazari ${ }^{1}$ \\ ${ }^{1}$ Shahrood University of Technology
}

November 3, 2020

\begin{abstract}
Today, immunotherapy is one of the effective treatments along with chemotherapy. This is especially true in cancers, which cause the immune system's failure. As a valuable tool, a mathematical model is developed in the form of ordinary differential equations (ODEs) to show the immune-tumor cells interaction. The macrophages polarization and cytokine dynamics are also regarded using standard pharmacokinetics-pharmacodynamics assumptions for better presenting the tumor-immune cells interaction. Its lack in researches of chemo-immunotherapy of cancer is what motivated us to come with an ODE model. The positivity of solutions, the equilibrium points, and their stabilities are studied. Moreover, the sensitivity of the final tumor population and response time to initial conditions and parameters' values are investigated. Initial conditions of the adaptive immune system and the parameters related to tumor cells proliferation, tumor carrying capacity, and exponent of fractional tumor cell kill by $\mathrm{CD} 8+\mathrm{T}$ cells have more effect on the final tumor population and the response time of tumor growth. Then, numerical simulations for pulsed chemotherapy, pulsed immunotherapy, and chemo-immunotherapy are presented and compared. Three patients with different immune responses are regarded to show the effect of immune response on treatment strategy. Numerical simulations show the effectiveness of the immunotherapy along with chemotherapy for cancer treatment. Also, it is shown that the immune system's strength has a remarkable role in cancer cure.
\end{abstract}

\section{Hosted file}

Manuscript_Mathematical Methods in the Applied Sciences.pdf available at https://authorea. com/users/372499/articles/490516-a-mathematical-model-for-chemo-immunotherapy-of-cancerconsidering-macrophages-polarization-and-cytokine-dynamics

\section{Hosted file}

ManuscriptFigures_Mathematical Methods in the Applied Sciences.pdf available at https:// authorea.com/users/372499/articles/490516-a-mathematical-model-for-chemo-immunotherapyof-cancer-considering-macrophages-polarization-and-cytokine-dynamics 